\title{
Le Pétrarque des antipétrarquistes français des
} années 1550

L'amour pris au tragique

Jean-Yves Vialleton

\section{CpenEdition}

\section{Journals}

Édition électronique

URL : http://journals.openedition.org/cei/619

DOI : $10.4000 /$ cei.619

ISSN : 2260-779X

Éditeur

UGA Éditions/Université Grenoble Alpes

Édition imprimée

Date de publication : 15 avril 2006

Pagination : $99-115$

ISBN : 978-2-84310-081-9

ISSN : $1770-9571$

Référence électronique

Jean-Yves Vialleton, «Le Pétrarque des antipétrarquistes français des années 1550 », Cahiers d'études italiennes [En ligne], 4 | 2006, mis en ligne le 15 octobre 2007, consulté le 10 décembre 2020. URL http://journals.openedition.org/cei/619 ; DOI : https://doi.org/10.4000/cei.619 


\title{
LE PÉTRARQUE \\ DES ANTIPÉTRARQUISTES FRANÇAIS DES ANNÉES 1550
}

\author{
L'AMOUR PRIS AU TRAGIQUE
}

Jean-Yves Vialleton

Université Stendhal Grenoble 3

Dans L'École des femmes, une «vieille charitable» explique l'amour à Agnès (II, 5). Horace est blessé, mais ce n'est pas qu'Agnès a fait choir quelque objet sur lui, comme elle le croit, c'est qu'elle a dans les yeux de quoi causer le trépas, un "venin " qui peut tuer un jeune homme. Ce «venin" peutil être considéré comme la parodie d'un trait pétrarquiste? On pense certes à «la poison amère» qui sort des «beaux yeux» dans les poèmes de Ronsard, au dolce veneno de Pétrarque, mais l'image de l'œil empoisonneur est déjà dans Ovide. Peut-être alors faut-il pour parler de pétrarquisme à propos d'un texte faire le constat d'une "réunion de traits"? C'est ce que propose par exemple A. Gendre, qui livre une liste pédagogiquement bien utile des motifs et figures du pétrarquisme ${ }^{1}$. D'autres études insistent moins sur le code pétrarquiste que sur ses variations et visent à caractériser le pétrarquisme de telle ou telle littérature, de tel ou tel auteur ("Le pétrarquisme de...») 2. J. Rousset a pu ainsi proposer une belle opposition entre un pétrarquisme blanc et un pétrarquisme noir.

En somme, d'un côté on est amené à reconstituer le modèle du poème pétrarquiste, comme Viollet-le-Duc reconstituait pour un monument à restaurer un "état complet qui peut n'avoir jamais existé», on dresse une sorte de mode d'emploi pour qui ne voudrait écrire qu'un pastiche de

1. André GENDRE, «Vade mecum sur le pétrarquisme français», Versants, 7 (1985), p. 37-65; on en trouve un résumé dans son édition des Amours de Ronsard: RONSARD, Les Amours et Les folâtries (1552-1560), Paris, LGF (Le Livre de Poche classique), 1993, p. 10-12.

2. Voir par exemple Arnaldo Pizzorusso, «Il petrarchismo di Desportes", Studi petrarcheschi, V (1952), p. 237-297, ou Giselle MATHIEU-CASTELLANI, Les Thèmes amoureux dans la poésie française, 1570-1600, Paris, Klincksieck, 1975, p. 32-72 et p. 219-232. 


\section{JeAn-Yves Vialleton}

Pétrarque. De l'autre, on vise l'écart plutôt que le type, mais alors le pétrarquisme apparaît moins comme l'imitation du Canzoniere que comme une création collective européenne, au même titre que la cathédrale gothique ou l'orchestre symphonique. C'est d'ailleurs ce à quoi nous invite J. Vianey qui ouvre son étude classique sur le pétrarquisme français par ce paradoxe:

Quand on étudie nos poètes pétrarquistes, on se borne le plus souvent à déve-

lopper cette affirmation: ils ont imité Pétrarque. Or, ce n'est là qu'une demivérité. Allons plus loin, c'est presque une erreur ${ }^{3}$.

J. Vianey montrait en effet l'importance dans les poèmes "pétrarquistes" des emprunts à des poètes italiens plus récents; par exemple, dans l'Olive, premier canzoniere en sonnets français (et non plus en dizains), Du Bellay n'emprunte à Pétrarque que huit ou dix sonnets et puise en revanche largement dans l'Arioste. J. Vianey proposait même une périodisation de la

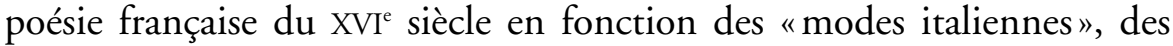
poètes successivement pillés.

On peut cependant échapper au dilemme de ces deux définitions du pétrarquisme en considérant celui-ci moins comme une "influence» de Pétrarque que comme une référence à Pétrarque. On pourra alors exclure des textes qui, bien que réunissant des traits présents dans Pétrarque, ne les font pas fonctionner comme référence (en particulier parce que ces traits y sont perçus comme étant tombés dans le domaine public). On pourra en revanche y intégrer des poèmes qui ne prennent sens que par cette référence, tout en n'étant pas des traductions, imitations ou adaptations de Pétrarque. C'est par exemple le cas de ce chant royal de Marot que cite Sébillet dans son Art poétique, où rien n'est pétrarquien, mais dont le dernier vers de chaque strophe traduit un vers de Pétrarque ${ }^{4}$. On pourra aussi justement intégrer au pétrarquisme français le fameux «antipétrarquisme» des années $1550^{5}$. Parler de référence à Pétrarque évite aussi de réduire le point de vue à l'examen de la réécriture du Canzoniere (avec ses si nombreuses modalités qu'elles pourraient sûrement rendre fou un théoricien de l'«intertextualité» à la recherche d'une taxinomie) et per-

3. Joseph VIAnEY, Le Pétrarquisme en France au XVI siècle, Montpellier, Coulet et fils, 1909, p. 6.

4. Thomas SÉBILlET, Art poétique français, II, 5, in Traités de poétique et de rhétorique de la Renaissance, éd. Francis Goyet, Paris, LGF (Le livre de poche classique), 1990, p. 116-117 ("débander l'arc ne guérit point la plaie», repris d'un vers de Erano i capei d'oro, Canzoniere, 90).

5. On en trouve un commentaire dans François Rigolot, Poésie et Renaissance, Paris, Seuil, 2002, chapitre II : «Pétrarquisme et antipétrarquisme», p. 187-205. 
met de donner toute son importance à Pétrarque comme figure, comme personnage permettant de penser la littérature. Un des traits du pétrarquisme à ne pas négliger, c'est la manière dont s'y sont liés très tôt la figure du poète et le texte de l'œuvre, avec l'écriture de "vies» et la publication d'éditions commentées du Canzoniere. En France particulièrement, pour reprendre les expressions de J. Balsamo, le "modèle pétrarquiste" ne se comprend pas si l'on ne prend pas en compte le «mythe pétrarquien ${ }^{6}$.

Les quelques textes souvent cités avec plus ou moins de raison au chapitre de l'"antipétrarquisme» sont des textes de la seconde génération du pétrarquisme français, celle de la Pléiade, après l'«invention " politique de Pétrarque sous François I ${ }^{\text {er }}$ et avant le mouvement anti-italianiste avec lequel ils n'ont donc rien à voir ${ }^{7}$. C'est par ces textes qu'est attestée l'apparition du verbe pétrarquiser. On peut dégager à partir d'eux une définition "en creux» de ce qu'est le pétrarquisme en ce début des années 1550 et voir comment le personnage de Pétrarque y sert de «moment de la réflexion sur la littérature ${ }^{8}$.

\section{Ou Pétrarque ou rien}

Le Grand Robert (édition 2001) donne comme première attestation pour le verbe pétrarquiser "vers 1555 " et donne comme première acception «Aimer platoniquement (comme Pétrarque aimait Laure)» en l'illustrant par l'Odelette à sa maîtresse de Ronsard.

Cette pièce des Mélanges de Ronsard, non reprise dans l'édition des œuvres de 1560, est traditionnellement rangée parmi les poèmes antipétrarquistes. Elle refuse en effet l'amour pour une femme chaste et cruelle:

Je veux aymer ardentement

Aussi veus-je qu'egallement

On m'ayme d'une amour ardente. (v. 1-3) 9

6. Jean BALSAMO, «Mythe pétrarquien et modèle pétrarquiste en France au XVI siècle», dans L'Italia lettteraria e l'Europa, ed. Nino Borsellino, Rome, 2001, p. 109-128. Le «culte de Pétrarque "(1533-1552) sert au prestige de François I ${ }^{\text {er }}$, qui se posait ainsi en nouveau Laurent de Médicis. On doit à Jean Balsamo, outre divers autres articles sur le sujet, la synthèse «Le pétrarquisme en France au XVI' siècle», dans l'édition revue du Dictionnaire des lettres françaises. XVIT siècle, LGF (La Pochothèque), p. 931-939.

7. Jean BALSAMO, Les Rencontres des Muses. Italianisme et anti-italianisme dans les lettres françaises de la fin du XVI siècle, Genève, Slatkine, 1992.

8. L'expression est empruntée à Graziano ARRIGHetTI, Poeti, eruditi e biografi. Momenti della riflessione dei Greci sulla letteratura, Pise, Giardini, 1987.

9. Ronsard, Euvres complètes, éd. G. Cohen, Paris, Gallimard, 1950, t. II, p. 797. 
Mais ce que Ronsard reproche à ceux qui pétrarquisent, c'est d'abord une erreur esthétique, une faute concernant la convenance entre le style et la «nature» du sujet, des propos ridicules parce que hors de saison: ils écrivent froidement alors que l'amour est ardeur, ardeur toute pétrarquienne où l'on reconnaît le fervor, l'échauffement sanguin de la tradition:

Les amans si frois en esté,

Admirateurs de chasteté,

Et qui morfondus petrarquisent,

Sont toujours sots, car ils meprisent

Amour qui de sa nature est

Ardent et pront, et à qui plest

De faire qu'une amitié dure

Quand elle tient de sa nature. (v. 9-16)

Le reproche a des conséquences éthiques, ceux qui pétrarquisent en jouant les dissimulateurs s'avèrent être des simulateurs:

Toute amitié froidement lente,

Qui peut dissimuler son bien

Ou taire son mal, ne vaut rien (v. 4-6)

On reconnaît ici moins une attaque contre Pétrarque que contre ceux qui le copient au lieu de l'imiter. Cette accusation contre les pétrarquistes conçus comme voleurs et faux poètes est un thème déjà présent dans la poésie italienne: on le trouve chez Nicolas Franco dès les années $1530^{10}$. Comme chez Ronsard, on reproche à ceux qui pétrarquisent de manquer $\mathrm{du}$ feu de l'amour. En somme, il faut distinguer «bien pétrarquiser» et "mal pétrarquiser».

Ce qu'est "bien pétrarquiser", Ronsard l'a défini dans une Élégie à Cassandre («Mon œil, mon cœur...»), publiée dans Le Bocage en 1554 et reprise dans le premier livre des Amours ${ }^{11}$. Dans ce texte, Ronsard regrette que son devoir de louer le roi l'oblige à abandonner la veine amoureuse. Les vers 39-43 énumèrent les erreurs de ceux qui n'ont pu «apprendre l'art de bien Petrarquiser» (v. 44), parce que ces mauvais poètes s'élèvent trop haut (ils tombent dans l'enflure, se soucient plus des «sentences» que des vers) ou descendent trop bas (ils sont "esnervés", c'est-à-dire sans muscle; vulgaires, ils peignent leur amie en "dame paillarde»). En somme, ils n'atteignent ni le grand style ni le vrai style simple. Quand Ronsard fait la liste des auteurs sur lesquels il a travaillé pour préparer ses

10. VIANEY, Le Pétrarquisme en France..., cit., p. 171, renvoie à un article d'Arturo Graf.

11. Ronsard, Euvres complètes, éd. citée, t. I, p. 98-100. 
poèmes amoureux (v. 13-15), il met d'un côté "Catulle, / Ovide, et Galle, et Properce et Tibulle», de l'autre Pétrarque. On note que "Galle» est Gallus, l'auteur d'élégies perdues: on voit que parmi les auteurs que Ronsard donne comme des lectures comptent moins en fait les textes euxmêmes que l'idéal que le nom désigne. La liste semble opposer les antiques et le moderne, ou la poésie de langue savante et celle de langue "vulgaire ${ }^{12}$, et aussi le genre de l'élégie et la poésie de Pétrarque. Les lectures de Ronsard le préparent à décliner tous les styles. Ronsard dit avoir dû laisser en chantier (v. 25-26)

Mainte Élégie à la façon antique,

Mainte belle Ode, et mainte Bucolique

qui auraient pu montrer que la France autant que la Toscane était heureuse

A soupirer une plainte amoureuse.

Pétrarque est donc à la fois une partie de la poésie amoureuse, celle qui s'oppose aux élégiaques antiques ou néo-latins, et le tout de la poésie, le modèle à égaler dans la poésie amoureuse, mais par cette «imitation » prônée par la Pléiade, qui n'est pas imitation au sens moderne, mais volonté de rivaliser en excellence au-delà même des différents styles possibles.

Le verbe pétrarquiser apparaît avant 1555, dans un texte de Du Bellay, À une dame, poème ajouté dans la réédition de 1553 d'un recueil de 1549 dédié à Marguerite de Valois ${ }^{13}$. Le Grand Robert cite le texte pour illustrer pétrarquiser au sens de "Imiter Pétrarque; chanter les perfections de sa maîtresse par des comparaisons outrées et précieuses qui sentent l'artifice». Il ne retient pas le texte comme première attestation du verbe, car il le date de 1558. C'est à cette date en effet que le poème modifié est republié dans Divers jeux rustiques et autres euvres poétiques sous le titre «Contre les Pétrarquistes» (ce qui permet au dictionnaire de dater l'apparition de ce dernier mot). Le texte est lui aussi cité pour illustrer l'antipétrarquisme et à ce titre rapproché de l'Odelette à sa maîtresse de Ronsard ${ }^{14}$.

12. La liste connaît des variantes: de 1567 à 1573, «Ovide, et Galle» est devenu «Marulle, Ovide». De fait, le poète moderne néo-latin Marulle est la source principale selon Belleau luimême, auteur du commentaire des secondes Amours ("Tout est pris de Marulle»). De 1578 à 1587, une nouvelle correction inverse les mots à la rime et insiste sur la science de Catulle («Tibulle / Properce, Ovide et le docte Catulle»).

13. Joachim Du Bellay, Euvres poétiques, éd. H. Chamard, Paris, Hachette, STFM, t. IV, 1919 , p. 205-215 (pour le texte de 1553; le texte de 1558 se trouve au tome V).

14. Voir par exemple RonSARD, Euvres complètes, éd. cit., t. II, note de la p. 797, p. 1115. 


\section{Jean-Yves Vialleton}

Le poème de $\mathrm{Du}$ Bellay relève du genre de la palinodie. Le poète explique qu'il refuse dorénavant le discours amoureux et ses artifices au profit d'une écriture de la sincérité, mais dans la dernière des trente strophes il se dit prêt à tenir ce discours si telle est la volonté de la dame. Le poème commence par des vers souvent cités:

J'ay oublié l'art de petrarquizer.

Je veulx d'amour franchement deviser

Sans vous flater, \& sans me deguiser.

Ceulx qui font tant de plaintes,

N'ont le quart d'une vraye amytié,

Et n'ont pas tant de peine la moitié,

Comme leurs yeulx, pour vous faire pitié,

Getent de larmes feintes. (v. 1-8)

Le développement est fondé sur la revendication d'un discours assurant sans obstacle la transparence de la communication (v. 2-3). La dénonciation de l'artifice du discours amoureux y reprend les lieux de la dénonciation de la rhétorique présentée comme sophistique. On trouve dès le début la critique attendue dans ce cadre de la figure de l'amplificatio qui rend plus grand de la moitié ou des trois quarts (v. 5-6). Dans la strophe 27, le discours amoureux est assimilé à une "belle harangue". Cette rhétorique attaquée, c'est d'abord celle du grand style: la dame y devient une déesse (strophe 7), on mobilise la mythologie (strophe 3). C'est la rhétorique des émotions fortes, du movere: la première strophe concerne la pitié, la seconde la frayeur (feu et horreur, orage, flamme, glaçon, flèches, liens et autres "semblables oultraiges»). Sont inventoriés les éléments de terribilità traditionnels: "ascendant" stellaire (strophe 6), «eternelles nuicts» et "horreur inhumaine» (strophe 8), héros mythologiques maudits (Prométhée, Tantale, strophe 14). Mais le poème a une visée encyclopédique et c'est à l'ensemble des styles possibles que Du Bellay s'en prend. La strophe 3 évoque la préciosité de la rhétorique du delectare (fin or, perles, cristal, marbre, ivoire), la strophe 5 la rhétorique de la copia (richesse, abondance), l'asianisme, le style fleuri, la rhétorique des peintures de la seconde sophistique:

Tout l'orient avec toutes les fleurs

Dont le printemps bigarre ses couleurs,

$\mathrm{Ne}$ fourniroient à peindre voz valeurs

Ny le cor d'Amalthée.

Ce refus des styles haut et moyen n'est pas au service d'une revendication du style simple. La strophe 23 introduit certes un ton comique lié au motif de la dénonciation de la folie universelle ("Je rys souvent, voyant 
pleurer ces foulx») et de la santé («je veux sain $\&$ dispos demeurer»). Mais il s'agit bien de congédier tous les styles possibles, ce que soulignent les généralisations et énumérations des strophes 15 à 18. La strophe 15 évoque les divers choix contradictoires ("l'un vole bas, \& l'autre vole hault»), la strophe suivante se moque de la volonté de les faire s'épouser dans une concordia discors. La strophe 18 rappelle la partition traditionnelle en trois styles:

Cestui voulant plus simplement aimer

Veult un Properce \& Ovide exprimer,

Et vouldroit bien encor' se transformer En l'esprit d'un Tibulle.

Mais cestui la comme un Petrarque ardent

Va son amour \& son style fardant.

Cet autre encor' va le sien mignardant Comme un autre Catulle.

Le dolce stil novo avait lui aussi en son temps refusé la rhétorique au profit de la sincérité. Voilà maintenant que Pétrarque retrouve sa place dans la rhétorique, et dans la partition des styles amoureux, il est nettement du côté du style du movere; l'épithète de nature dont il est pourvu ("ardent») le range comme figure de l'écriture du fervor et comme représentant du style véhément opposé aux styles simple et moyen des élégiaques antiques.

Dans l'avertissement "Au lecteur" des Quatre premiers livres des Odes en 1550, Ronsard utilise, plus tôt encore, le verbe pétrarquiser, au participe passé. Il y oppose le "style à part» qu'il a choisi sur le modèle de Pindare et d'Horace et celui goûté par les «rimeurs [c'est-à-dire les faux poètes] et principalement les courtizans" "qui n'admirent qu'un petit sonnet Petrarquizé, ou quelque mignardise d'amour qui continue toujours en son propos " ${ }^{15}$. L'année précédente, dans La Défense et Illustration de la langue française, Du Bellay trouve encore le sonnet une invention "non moins docte que plaisante». Mais très vite la Pléiade oublie ce que Pétrarque pouvait avoir de mondain ou de plaisant. Elle semble ignorer la piacevolezza de Pétrarque au profit de sa seule gravitas. Pétrarque, c'est le grand style. La simplification de Pétrarque par le pétrarquisme français en découle. L'emploi du décasyllabe, on le sait, participe de cette recherche de l' "héroïque» ${ }^{16}$. Le sonnet devient la forme obligée, parce que, en ce début des années 1550 , c'est une forme nouvelle propre à remplir une case

15. RONSARD, Euvres complètes, éd. cit., t. II, p. 973.

16. Yvonne BELLANGER, «Ronsard et le décasyllabe», Bibliothèque d'Humanisme et Renaissance, XLIV, (1982), p. 493-520. 


\section{Jean-Yves Vialleton}

vide, une forme qui seule permet d'unir les contraires, c'est le seul «petit» poème par sa brièveté qui est une "grande forme" par sa "gravité», comme l'expliquent les arts poétiques ${ }^{17}$.

Celui qui imite Pétrarque est associé dans le poème de Du Bellay $\grave{A}$ une dame au "fard» qui depuis Platon est celui de la sophistique. Mais sont aussi blâmés ceux qui tendent aux styles simple et moyen. Le premier vers du poème, souvent cité, semble créer une opposition entre un pétrarquisme menteur et le projet d'une écriture sincère refusant l'artifice. Mais cette opposition n'est pas celle du style élevé et du style simple. Une ambiguïté caractérise le texte, qui est analogue à celle qu'on trouve dans l'Élégie à Cassandre.

Cette ambiguïté ne doit pas étonner, car elle est celle qui caractérise le grand style dans la tradition rhétorique cicéronienne, grand style conçu comme un style "récapitulatif» (Orator $\$ 99$ ), à la fois un des styles et celui qui les englobe tous, selon un fondement anthropologique bien connu $^{18}$. Jean Lecointe a montré comment cette notion avait modelé à la Renaissance l'idée du grand auteur ${ }^{19}$. C'est ainsi que fonctionne déjà l'image de Pétrarque dans la Défense et Illustration de la langue française, comme l'a mis en évidence Francis Goyet dans son commentaire ${ }^{20}$.

Dans la version de 1558 du poème de Du Bellay, certaines strophes sont supprimées, d'autres changent de place, quelques modifications sont apportées au texte. Les deux modifications les plus significatives sont évidemment le changement de titre et un changement de trois syllabes du premier vers de la dernière strophe: "Si toutefois tel style vous plaît mieux» est remplacé par «Si toutefois Pétrarque vous plaît mieux». Cette seconde version semble plus nettement antipétrarquiste, mais elle ne le semble que dans la mesure même où Pétrarque est plus nettement encore élevé jusqu’à incarner la poésie elle-même. Si Du Bellay écrit «contre les

17. Sébillet (1548) assimile le sonnet à l'épigramme italien et au dizain français, mais souligne qu'il se distingue par son contenu et le rattache à Pétrarque: «la matière facétieuse est répugnante à la gravité du sonnet qui reçoit plus proprement affections et passions graves, même chez le Prince des poètes italiens, duquel l'archétype des sonnets est tiré» (chapitre II, in Traités de poétique et de rhétorique de la Renaissance, cit., p. 105). Jacques Pelletier dans son Art poétique (1555) le range entre l'ode et l'épigramme, mais en précisant que «le sonnet est plus hautain que l'épigramme, a plus de majesté, et est capable de discours grave, mais qui soit bref» (II, 4, in Traités de poétique et de rhétorique de la Renaissance, cit., p. 270).

18. C'est l'«enveloppement des contraires " par celui des termes qui est réputé supérieur tel que le décrit Louis DumONT dans Homo hierarchicus: le système des castes et ses implications, Paris, Gallimard, 1970.

19. Jean LECOINTE, L'Idéal et la Différence. La perception de la personnalité littéraire à la Renaissance, Genève, Droz, 1993.

20. Joachim Du Bellay, Euvres complètes, Paris, Champion, 2003, t. I, p. 263 et p. 361-362. 
pétrarquistes", c'est que son poème se fonde sur le paradoxe du discours amoureux qui refuse le discours amoureux, qu'il ne se développe que par une sorte de prétérition généralisée par laquelle se fait l'inventaire encyclopédique des différents discours amoureux. De la rhétorique, Du Bellay ne veut garder que l'expression de l'éthos fondée sur l'utopie d'une communication transparente ne pouvant aboutir sans prétérition qu'à l'absence de poème. Le pétrarquisme y apparaît à la fois un des styles possibles et en même temps désigne dans sa totalité les styles refusés par le poète. Pétrarque est une partie de la poésie et en même temps le tout de la poésie.

Bien plus, Pétrarque y apparaît même comme ce que les anthropologues à propos des mythes appellent un "héros culturel». La strophe 20 est une revendication de primitivisme qui développe un petit mythe de la chute qui est aussi un conte étiologique racontant le passage de la simple conversation de tous les jours à la poésie, dans lequel Pétrarque est une sorte de "décepteur", un diable étranger qui met fin à la communication transparente, mais aussi l'inventeur de la nouvelle science qu'est le discours amoureux:

\footnotetext{
Nos bons ayeux, qui cet art demenoient,

Pour en causer, Petrarque n'apprenoient,

Ains franchement leur dame entretenoient Sans fard ou couverture

Mais aussi tost qu'Amour s'est fait scavant,

Lui qui estoit François au paravant,

Est devenu menteur \& decevant, Et de Thusque nature.
}

Le thème du poème de Du Bellay n'est pas nouveau : des rapprochements ont été faits avec des textes de Saint-Gelays et de Jodelle. Dans un fragment d'un poème de danse (une chanson en trois branles), Jodelle joue déjà sur une parole amoureuse qui signe sa vérité sur le refus du discours amoureux $^{21}$. Le poème fait l'éloge de l' "amour simple» (strophe 23), mais lui non plus ne refuse pas le grand style au profit du style simple. Il attaque le style enflé des capitans de l'amour, mais c'est parce que ce style ne fait pas sentir que l'âme est vraiment malade (strophe 17). La «fable» est refusée, mais seulement parce que la réalité de l'amour du poète dépasse la fiction (refrain 2)! Le poème de Du Bellay comme celui de Jodelle relève de l'éloge paradoxal du refus de la poésie. La logique de ce paradoxe, c'est le refus de l'expression: 
Ma passion qui a peur Qu'on la juge feinte,

Veut se couvrir dans le cœur Sans s'ouvrir par plainte ${ }^{22}$.

Mais, comme l'objecte Ronsard, que vaut la poésie de celui qui « dissimule son bien" et "tait son mal»? La poésie, c'est tout ou rien. C'est Pétrarque ou rien.

\section{L'amour comme comédie, l'amour comme tragédie}

On sait que Ronsard dans ses Amours s'est livré à un exercice de style. Les Amours de Cassandre sont son canzoniere conforme au style élevé attribué à Pétrarque, mais elles sont suivies de deux Continuations qui empruntent avec ostentation une toute autre voie. Les poèmes pour Marie s'y donnent comme un refus de Pétrarque au profit de la simplicité des élégiaques antiques, mais, on l'a souvent noté, ces poèmes antipétrarquistes sont à bien des égards tout aussi pétrarquiens et parfois plus encore. Ronsard par exemple abandonne un "beau nom» (selon l'expression de Claude Binet dans sa biographie de Ronsard) de l'aristocratie italienne qui est aussi un nom homérique de prophétesse pour un nom français tout simple, mais c'est pour mieux retrouver un nom avec cinq lettres comme celui de la Laura, ou encore de la Délie de Scève.

La conjonction pétrarquienne de l'homme et de l'œuvre que Ronsard adopte (il fait faire des commentaires à ses Amours, avec notations biographiques) pose un épineux problème de persona quand on veut faire plusieurs canzonieri successifs. Pour résoudre ce problème, Ronsard va faire de Pétrarque lui-même un personnage dans un poème qui est une manière de postface puis de préface: sous le titre $\grave{A}$ son livre, c'est le dernier des soixante et un poèmes de la Nouvelle continuation, sous le titre Élégie à son livre, le poème qui ouvre en 1560 Le second livre des amours ${ }^{23}$. Le poème est une apostrophe au livre personnifié en fils qui s'en va (v. 1-32). Le poète lui indique ce qu'il doit répondre à «quelque dame honnête et gentille de cœur » qui blâmerait Ronsard de son inconstance amoureuse,

Dequoy je ne devois abandonner Cassandre,

Qui la premiere au cœur le trait d'Amour me meist,

22. Étienne Jodelle, Euvres, ed. cit., p. 320.

23. Ronsard, Les Amours et Les folatries (1552-1560), éd. A. Gendre, Paris, LGF (Le Livre de Poche classique), 1993, p. 450-458. 
Et que le bon Petrarque un tel peché ne feist,

Qui fut trente $\&$ un ans amoureux de sa dame,

Sans qu'une autre jamais luy peust eschaufer l'ame. (v. 36-40)

Cette réponse se présente comme un portrait burlesque de Pétrarque:

Luy mesme ne fut tel: car à voir son escrit

Il estoit esveillé d'un trop gentil esprit

Pour estre sot trente ans, abusant sa jeunesse,

Et sa Muse, au giron d'une seule maîtresse:

Ou bien il jouissoit de sa Laurette, ou bien

Il estoit un grand fat d'aymer sans avoir rien,

Ce que je ne puis croire [...] (v. 45-51)

Ronsard cite les épithètes «chaste, divine, sainte» en en faisant de simples compliments de gratitude de l'amant satisfait par sa maîtresse. Il poursuit en affirmant que si l'amante devient "de pis en pis tousjours" (v. 61), il faut l'abandonner «sans se rompre la teste/De vouloir adoucir une si sotte beste» (v. 63-64). Il attaque les femmes qui se laissent trop attendre et forcent à dépenser en repas et musique, à se risquer à des duels. Leur chasteté n'est en fait qu'une ruse.

La citation de Pétrarque, comme la qualification de la lectrice offensée, renvoie à une célèbre énumération d'épithètes d'un poème de Pétrarque: "Gentile,/santa, saggia, leggiadra, honnesta e bella". Cette énumération figure à la fin du premier quatrain du poème $247 \mathrm{du}$ Canzoniere, sonnet dans lequel Pétrarque répond justement à ceux qui pourraient juger excessif le style avec lequel il loue Laura.

Les trente et un derniers vers du poème $\grave{A}$ son livre sont un art poétique. À ceux qui le blâment d'avoir abandonné le style élevé ("grave", gravis; «bouche magnifique») de ses odes ("humeur pindarique»), il faut répondre par un argument qui concerne la convenance du style au sujet:

Dy luy que les amours ne se souspirent pas

D'un vers hautement grave, ains d'un beau stille bas,

Populaire \& plaisant, ainsi qu'a fait Tibulle,

L'ingénieux Ovide, \& le docte Catulle:

Le fils de Vénus hait ces ostentations:

Il sufist qu'on luy chante au vray ses passions,

Sans enfleure ny fard, d'un mignard \& doux stille

Coulant d'un petit bruit comme une eau qui distille. (v. 173-180)

On retrouve les adjectifs et images que la tradition associe à la définition du style simple, comme "beau style bas». On retrouve également le nom des élégiaques latins. Le nom d'Ovide permet de renvoyer à l'ouverture des Amours de celui-ci (I, 1), où l'inconstance est liée à la question du 


\section{Jean-Yves Vialleton}

genre (passage de l'épopée à la poésie amoureuse) elle-même liée à celle de la forme qui convient (le pentamètre à la place de l'hexamètre). Manque le nom de Properce, mais celui-ci est évoqué par le texte lui-même qui reprend un de ses traits ("carmina mansuetus lenia quaerit Amor») ${ }^{24}$.

Cependant Ronsard ne reprend pas l'opposition entre élégie et épopée; à l'élégie il oppose la tragédie:

S'il avient quelque jour que d'une voix hardie

J'anime l'eschaufaut par une tragedie

Sententieuse \& grave, alors je feray voir

Combien peuvent les nerfs de mon petit sçavoir:

Et si quelque Furie en mes vers je rencontre,

Hardi, j'opposeray mes Muses alencontre,

Et feray resonner d'un haut \& grave son

(Pour avoir part au bouc) la tragique tansson:

Mais ores que d'Amour les passions je pousse,

Humble, je veux user d'une Muse plus douce. (v. 183-192)

Dans le poème déjà cité de Jodelle le style haut était déjà incarné par le style tragique:

Mesme pour tragiquer mieux,

Ils recourent furieux

La cité, race, et maison

Thebaine ou Troyenne. (II, 10) ${ }^{25}$

Ce parallèle entre l'élégie et la tragédie se trouve déjà chez Ovide (Amours III, 1). Mais le couple dissymétrique ne peut que faire surgir l'élément attendu absent, la comédie. Ovide lui-même avait fait entrer le thème de la comédie dans l'élégie amoureuse. Le style simple des Continuations est mis sous le signe des élégiaques latins, la référence est explicite et on en a souvent expliqué les enjeux après Paul Laumonier ${ }^{26}$. Mais le parallèle avec la tragédie suggère que le sujet du comique de Ronsard mériterait probablement quelque attention, d'autant qu'entre les premières Amours et les Continuations est publié le Livret des folâtries.

Malgré ce qu'on pourrait croire, la première Continuation et les scandaleuses Folatries ont bien des points communs: la référence à Catulle, la conclusion par des «épigrammes grecques"... Surtout les Folâtries et les deux Continuations sont mises sous le signe de Bacchus. Après les pre-

24. Élegies I, 9, 12 «L'Amour est douceur et veut de tendres accents» (ProperCE, Élégies, texte et trad. de D. Paganelli, Paris, Les Belles Lettres, 1929).

25. JODElle, Euvres, éd. cit., t. I, p. 331.

26. Sur le modèle catullien dans les Amours, voir LECOINTE, L'Idéal et la Différence..., cit., p. 541. 
mières Amours, on passe de la "guerrière" aux jeunes angevines, du ciel à la terre, mais aussi d'Apollon à Bacchus. À l'«arbrisseau " de Phébus, laurier prophétique ou lauro toscan, s'ajoute le lierre dionysiaque (Folâtries, "Dithyrambes», v. 159; Continuation, 58 et 59, «Il ne sera jamais [...] ; , "J'aurais toujours au cœur [...]») et le pin de Cybèle. Bacchus est "beaucoupformes", mais c'est bien sûr le dieu du théâtre. Les références à la tragédie sont explicites dans les textes parlant de Bacchus. Le cadre campagnard est celui du «bocage" pastoral, mais c'est aussi le lieu d'origine du théâtre, né de la campagne et particulièrement selon Evanthius des fêtes de la vigne. Une des étymologies de comæedia rattache le mot à un mot grec signifiant le village. Une autre, présente chez Donat et Diomède, le rattache à comessari, "aller en chantant dans les villages les jours de fête», de comedere, "manger». Une définition médiévale courante était "chant de fête villageoise", "villanus cantus", "carmina quae in conviviis canuntur».

Dans les Folâtries, nombreuses sont, mêlées à mille antiquités, les allu-

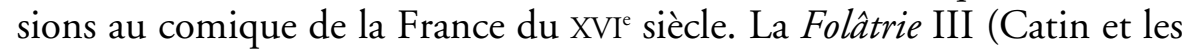
religieux paillards) met en scène des personnages de farce. A l'antique transe dionysiaque se superposent les jeux de "folie» (Dithyrambes, v. 206: «Il me plaist ores d'estre fol»), l'ivrognerie des fêtes "folkloriques" (Folâtrie VIII, 1: «le jour de sainct-Martin»). Le dithyrambe est la trace écrite de la bacchanale d'Arcueil, la "pompe" y était une sorte de charivari néo-antique organisé lors du Carnaval de 1553.

L'onomastique des Folâtries, prénoms ou diminutifs français, renvoie au genre comique ; c'est l'onomastique de la farce et celle qu'utiliseront certains traducteurs de comédies à la place ou en même temps que les noms gréco-latins et italiens. La manière de nommer le dédicataire dans l'épître initiale ("À Janot parisien») le range parmi les «messieurs tout le monde", les "humiles atque privatae personae» qui caractérisent selon Diomède (Ars grammatica III) la comédie, son "style comique et privé» (Montaigne, Essais I, 40). Les Continuations jouent souvent de la rhétorique de la douce jouissance, du delectare; elles relèvent plus du style fleuri que de la simplicité brutale des "vers raillards». Leur saison préférée n'est pas l'automne fructueux et vineux, non plus qu'un été de feu et de glace, mais bien le printemps, ses oiseaux et ses fleurs. Elles ont cependant pour noyau un tout simple prénom français. La recomposition des Amours donne aux amours villageoises une fin tragique en 1578, reprenant sinon la gravité générale du moins l'organisation bi-partite du Chansonnier de Pétrarque. Avaient été supprimées dès 1560 quelques pièces lestes de 1555, dont certaines étaient d'anciennes «folâtries». Mais la comédie pas- 


\section{Jean-Yves Vialleton}

torale est présente par l'introduction du "Voyage de Tours", avec son onomastique et sa toponymie françaises, ses fêtes "folkloriques" (les Mais, la "noce», la Saint Jean), ses poètes travestis sous des prénoms de farce (Thoinet et Perrot), son allusion finale au "pasteur Janot». C'est déjà la fête au village du Francion de Sorel, Les Vendanges de Suresnes de Du Ryer ou l'acte II de Dom Juan. L'importance nouvelle des «stratégies dialogiques " ${ }^{27}$ dans les Amours de Marie est peut-être aussi à mettre au compte de la référence à la comédie, dans un siècle où "comédie" était un mot possible pour titrer une traduction d'un «colloque» d'Érasme $(1541)^{28}$.

Si s'allient si bien le pastoral et le comique, c'est que tous deux s'opposent à l'héroïque et au tragique. La pastorale est le temps hors de l'Histoire, celui d'avant les héros et d'avant la tragédie, celui de l'enfance du monde et de l'adolescence d'Hippolyte avant l'amour de Phèdre. Le comique est le temps d'après, celui d'aujourd'hui. Pastorale et comique sont deux manières de concevoir le non-héroïque, deux manières d'incarner le style simple. On aurait tort de réduire l'imaginaire de la simplicité à son versant virgilien qui l'assimile à la nudité d'un lyrisme primitif. Le style simple, c'est aussi selon la tradition rhétorique le style de la convivialité familière (comitas) qu'a décrit Cicéron et après lui Quintilien, style qui se caractérise par le bon usage, le plaisant et l'ingénieux (De officiis I, 97104; Orator 75-90; De oratore II, 216-291; Institution oratoire VI, 2-3 et $\mathrm{X}, 1$ ), et dont l'un des modèles est Plaute. C'est au sein de cette seconde tradition du style simple que la théorisation de la «facétie» a trouvé sa place. La Renaissance italienne puis française est fascinée par ces facetiae et cette dicacitas du "style comique et privé», notions qui font se rencontrer, à propos de l'ingéniosité du bon mot, l'art de la conversation et celui de l'«épigramme raillard». Pétrarque, mais cette fois l'autre Pétrarque, l'auteur humaniste néo-latin, est là encore un avant-coureur de la Renaissance: il consacre à la facétie un chapitre du second livre des Rerum memorandarum libri. Comme l'a montré J. Vianey, bien des «gauloiseries » de la poésie française du XVI siècle sont empruntées au burlesque des poètes italiens ${ }^{29}$, comme la farce dite populaire au début du siècle suivant empruntera aux facezie de Poggio (ainsi en est-il du vieillard-poireau à la tête blanche et à la queue verte de Tabarin).

27. Michel JeAnNERET, «Les Amours de Marie: inscription de la deuxième personne et stratégies dialogiques", in Sur des vers de Ronsard (1585-1985), éd. M. Tetel, Paris, Aux amateurs de livres, 1990, p. 61-70.

28. Raymond LebĖGUE, "Tableau de la comédie française de la Renaissance», Bibliothèque d'humanisme et Renaissance, VIII (1946), p. 326.

29. Vianey, Le Pétrarquisme en France..., cit., p. 43. 
La mise en scène du "bon Pétrarque" par Ronsard dans l'art poétique des Amours de Marie est bien une mise en scène comique de Pétrarque. La "débauche» de la jeunesse et les "finesses» des femmes sont deux thèmes typiques du comique, présents dans le théâtre comique joué (les farces) plus encore que dans la définition savante laissée par la tradition de la comédie.

Quintilien (VI, 2) mettait la tragédie plutôt du côté du pathos, des émotions fortes propres à bouleverser (movere), la comédie plutôt du côté de l'éthos, de l'émotion douce, propre à construire le consensus (conciliare). Dans ses Continuations, Ronsard propose une version éthique, comique, "conciliatrice» de Pétrarque. Car Pétrarque, l'"ardent», le "transi", est un poète du pathos de l'amour, de la maladie d'amour, de ce qui deviendra la "passion" tout court. C'est ce que disent les textes de la Pléiade et cela est confirmé à la fin du siècle par Montaigne ${ }^{30}$. Dans le sonnet 11 de La Boétie rapporté dans les Essais (I, 9), les "amours douloureux» du «Florentin transi " s'opposent là encore à Catulle, le "folâtre» amoureux, et au "savant migrégeois Properce». Comme Jodelle et $\mathrm{Du}$ Bellay, La Boétie refuse la poésie amoureuse au nom de la vérité de l'amour, et plus nettement encore qu'eux, au nom de l'irréductible vérité du moi («Ils n'aiment pas pour moi, je n'aime pas pour eux»). Dans un autre chapitre des Essais, le chapitre De la tristesse (I, 2), qui est, on le sait, un chapitre contre la tristesse, écrit par un Montaigne qui se veut peu enclin aux «violentes passions», un vers de Pétrarque est cité pour illustrer les "passions insupportables».

Le pétrarquisme selon les poètes de la Pléiade peut donc se définir de façon simple. Pétrarque n'est pas seulement un auteur moderne à imiter parce qu'il a, en langue "vulgaire», réussi à égaler les Anciens. C'est aussi un auteur qui a traité le même sujet que les élégiaques antiques, mais qui l'a fait dans un registre nouveau. Les amours profanes, les erotica, sont traditionnellement un sujet comique: "amores, virginum raptus", voilà le sujet de la comédie. En somme, Pétrarque est celui qui a pris l'amour au sérieux, et plus précisément encore au tragique. Les définitions encore médiévales du tragique comme genre "héroïque et ensanglanté» énuméraient une liste de maux publics dont aucun n'est présent sinon comme métaphores dans le Canzoniere. Mais l'humanisme commence à élaborer une nouvelle définition de la tragédie, plus aristotélicienne, qui correspond parfaitement aux amours de Pétrarque: début heureux et fin

30. Jean Balsamo, article "Pétrarque», in Dictionnaire de Montaigne, éd. Ph. Desan, Paris, Honoré Champion, 2004, p. 780-781. 


\section{JeAn-Yves Vialleton}

funeste, recours massif aux figures, revirements perpétuels des passions, rhétorique visant fondamentalement la crainte et la pitié... En prenant l'amour au tragique, Pétrarque a occupé ce qui était une "case vide» du tableau de la littérature. On a pu dire, à cause du relatif effacement de l'œuvre de Pétrarque en latin, du Pétrarque chrétien et stoïcien ${ }^{31}$, à cause aussi de l'usage mondain du Canzoniere (offrir le livre était un cadeau amoureux de choix) que le Pétrarque français était un Pétrarque «laïque et galant» ${ }^{32}$. Mais l'expression rend mal compte du fait que ce Pétrarque français est aussi un Pétrarque tragique, qu'il autorise donc à donner aux simples jeux de l'amour profane une dimension morbide et sacrée, proche de la folie ("hôs idon, hôs énamen», "je la vis, je devins fou», est la devise en tête des premières Amours de Ronsard), une dimension philosophique par laquelle l'expérience "comique et privée» du trouble érotique se hausse au niveau des "fureurs héroïques", de ces "humeurs transcendantes» ou "fantastiques élévations" dont se moque Montaigne qui, comme Du Bellay dans le poème $\grave{A}$ une dame, préfere rester «sain et dispos».

Ce portrait de Pétrarque en poète tragique a permis aussi de structurer le champ du discours amoureux. Pétrarque montrait en fait dans son Chansonnier les nuances changeantes d'une même lumière. Ronsard propose lui des amours «décomposées» au prisme de la partition des styles et il multiplie ainsi les personae. En faisant succéder au solennel et douloureux amoureux de l'unique Cassandre la figure de l'amant inconstant, en particulier de l'amant gentiment railleur de Marie, Ronsard met en place un système de personnages, avec ses résonances philosophiques et physiologiques. La polarité de ses Amours sera celle qui organise l'Astrée, et les avatars de Céladon et d'Hylas se multiplieront. Des seconds amoureux "enjoués» doubleront les héros nobles et mélancoliques dans bien des romans du XVII siècle. Dans les comédies, par exemple dans la première comédie de Corneille, ils prendront même la première place, dénonceront les rhétoriques de l'amour, refuseront les plaintes et la mélancolie et prêcheront la soumission tranquille à l'ordre des choses.

Il y a cependant au moins un personnage qui échappe à ce système en miroir, c'est l'Agnès de L'École des femmes. Au discours amoureux rapporté par la «vieille charitable» fait pendant à l'acte suivant une lettre de la jeune fille (III, 4):

31. Franco SIMONE, «La fortuna del Petrarca nella prima metà del Cinquecento », in IDEM, $I l$ Rinascimento francese. Studi e ricerche, Turin, Società Editrice Internazionale, 1961, p. 141-222.

32. BALSAMO, "Le pétrarquisme en France au XVI ciècle», cit., p. 933. 
Je veux vous écrire, et je suis bien en peine par où je m'y prendrai. J'ai des pensées que je désirerais que vous sussiez; mais je ne sais comment faire pour vous les dire, et je me défie des paroles. Dis-moi franchement ce qui en est; car enfin, comme je suis sans malice, vous auriez le plus grand tort du monde, si vous me trompiez; et je pense que j’en mourrais de déplaisir.

Cette lettre en prose puérile ne refuse pas la poésie du cœur, mais elle le fait par un "degré zéro" de l'écriture poétique. Elle semble réaliser le rêve déjà rencontré dans les textes de Du Bellay, Jodelle et La Boétie, qui n'est pas le rêve d'un "langage des dieux", mais celui d'un langage humain rajeuni, retrouvant l'expression transparente des "bons aïeux", du cœur, ou du moi. La lettre d'Agnès, c'est la Poésie d'avant le Poème. Peut-on vraiment en rire? 